\title{
Langmuir probes in RF plasma: surprising validity of OML theory
}

\author{
Francis F. Chen
}

University of California, Los Angeles, CA 90024-1594

\begin{abstract}
Low-temperature, partially ionized plasmas are commonly used in industry for materials processing, and many of these are created by radiofrequency (RF) power. For the characterization of plasma sources, electrostatic probes are the easiest to use, but interpretation of the current-voltage $(I-V)$ curves is not straightforward. The presence of strong RF pickup and neutral collisions further complicates the problem. Langmuir's orbital-motionlimited (OML) theory provides a simple formula for ion current, but this is not expected to be valid in high-density plasmas. With carefully designed probes, it is found experimentally that OML can be used successfully under adverse conditions. Careful examination of the OML theory shows that its validity is fortuitous but can give correct results in commonly encountered conditions. The probe design, results, caveats, and methodology are given for use of probes in RF plasmas.
\end{abstract}

\section{Introduction}

Radiofrequency plasma sources used, for instance, in the semiconductor industry typically operate with plasma densities between $10^{10} \mathrm{~cm}^{-3}$ and $10^{12} \mathrm{~cm}^{-3}$. Langmuir probes used to determine density $n$ and electron temperature $T_{\mathrm{e}}$ in these plasmas suffer from two major complications: collisions of ions with neutrals while orbiting the probe, and the distortion of the probe's current-voltage $(I-V)$ characteristic by RF fluctuations in plasma potential. These fluctuations are caused by electron losses to the walls or electrodes at the RF frequency and are particularly strong in magnetized discharges, where potentials are carried along field lines. This paper describes experiments in which these effects can be minimized by proper design and application of the probes.

In a previous experiment ${ }^{1,2}$ in which we compared densities measured by microwave interferometry with those inferred from probe $(I-V)$ curves using various theories, it was found that these theories yielded ion densities $n_{\mathrm{i}}$ in error by as much as a factor of 3 to 5 . It was suggested that the discrepancy could be caused by charge-exchange collisions of the ions with neutrals as the ions spiraled into the probe. Such ions would then lose their angular momentum and be pulled radially into the probe by the sheath potential, giving rise to a current higher than predicted. In that work, it was found that the Allen-Boyd-Reynolds (ABR) theory, which neglects orbiting, underestimated $n_{\mathrm{i}}$ while the Bernstein-Rabinowitz-Laframboise (BRL) theory overestimated it. Langmuir's OML theory also gave an overestimate at low pressures but was found to be accurate at the highest pressure of $10 \mathrm{mTorr}$, a result that was unexplained.

The background of work on ion collection without collisions can be summarized as follows. OML assumes large sheaths such that $\xi_{\mathrm{p}} \equiv R_{\mathrm{p}} / \lambda_{\mathrm{D}}>>1$, where $R_{\mathrm{p}}$ is the probe radius and $\lambda_{\mathrm{D}}$ the Debye length. $\mathrm{ABR}^{3}$ allowed for finite sheaths but assumed radial ion motion only. Their results for spheres were extended to cylinders by Chen ${ }^{4}$. BRL started with a formulation by Bernstein ${ }^{5}$ allowing for both finite sheaths and orbiting for both spheres and cylinders, but he assumed monoenergetic ions. Laframboise ${ }^{6}$ extended this to Maxwellian distributions, requiring extensive computations. The resulting $I-V$ curves have been parametrized by $\mathrm{Chen}^{1}$. 
The effect of collisions on ion current was considered first by several authors in the continuum limit of short ion-neutral mean free path $\lambda_{\mathrm{m}}$. In 1966, Chou et al. $^{7}$ formulated a method to solve Laframboise's equations including collisions in a Krook term. This would include finite sheaths, orbiting, and collisions; but the mathematics and computations were so complicated that only spheres could be treated, and no useful $I-V$ curves were produced. in 1974, Zakrzewski and Kopiczynski ${ }^{8}$ used probes in a glow discharge with pressures between tens of mTorr and 1 Torr and found a peak in the ion current at intermediate pressures. They correctly surmised that the current first increased due to orbit interruption, and then decreased due to elastic scattering of the ions at high pressures. An ad hoc calculation of these two effects yielded curves agreeing with the data. In 1994, Tichý et al. ${ }^{9}$ combined the results of Refs. 7 and 8 to extend the validity range of the parameter $\lambda_{\mathrm{m}} / R_{\mathrm{p}}$. Their plot of argon ion current $v s . \lambda_{\mathrm{m}} / R_{\mathrm{p}}$ at fixed probe potential $V_{\mathrm{p}}$ showed clearly the peak at intermediate pressures and how it varies with $\xi_{\mathrm{p}}$. A Monte-Carlo computation by Trunec et al. ${ }^{10}$ showed graphically how the ion orbits were changed by collisions at low density, thus increasing the collected current. A graph of ion current vs. plasma density for $10^{7}<n<10^{10} \mathrm{~cm}^{-3}$ showed that the collision-induced error relative to OML was largest at $10^{7} \mathrm{~cm}^{-3}$ and decreased to zero around $10^{10} \mathrm{~cm}^{-3}$ for fixed $R_{\mathrm{p}}$ and $V_{\mathrm{p}}$. This was explained by the decrease in sheath thickness with $n$ : not many collisions can occur in a thin sheath.

This subject was revived by reports of the experiment of Evans et al. ${ }^{2,1}$. Sternovsky et al. ${ }^{11}$ calculated the current gained by charge exchange but did not solve for the revised $n(r)$ and $V(r)$ distributions, where $V(r)$ is the potential distribution, as did Laframboise and Chou et al. Instead, they relied on a proof from Liouville's equation that $n(r)$ is constant in a cylindrical sheath, with the increase in $n$ from collisional deflections just balancing the decrease due to acceleration. The results were compared with experiments in a low-density, low-pressure plasma with $n \leq 10^{7} \mathrm{~cm}^{-3}$ and Ar pressure $<1$ mTorr. They obtained ion currents agreeing with their theory but much larger than predicted by OML. The $I-V$ characteristic was linear, rather than parabolic, as in OML and observed in our work. However, the conditions were very different from those in this paper, the values of $\xi_{\mathrm{p}}$ being less than 0.1 . In a later paper ${ }^{12}, \xi_{\mathrm{p}}$ was increased to $\leq 0.49$ by increasing $R_{\mathrm{p}}$ and $n$, but still small compared with those in the present paper. At the highest $\xi_{\mathrm{p}}$ 's the predicted current was actually larger than that observed. The scattering of ions after orbit disruption would not be effective at these low pressures. In 2006, Iza and Lee ${ }^{13}$ did particle-in-cell simulations for a few million particles around a probe $0.2 \mathrm{~mm}$ in radius, in a 3 $\mathrm{eV}, 10^{9} \mathrm{~cm}^{-3}$ plasma in 1, 10, and 100 mTorr of Ar. They included collisions of all types and concentrated on the presheath and the floating potential. For ion currents, they found, as usual, the density predicted by the BRL and OML theories were too high, and that predicted by the ABR theory was too low. The theory of Tichý et al. fit their results best. Their $I-V$ curves were more parabolic than linear.

Interest in the floating potential of spheres arose from dusty plasma and space research, and papers have addressed this problem when there are collisions in the sheath. Lampe et al. ${ }^{14}$ were able to solve the problem analytically. Pletnev and Laframboise ${ }^{15}$ marshaled the whole computational scheme originally used by Laframboise and added Monte-Carlo solutions to add collisional effects, but only for $\mathrm{N}_{2}$. As expected, they obtained higher ion currents at low densities than in collisionless OML theory. However, the discrepancy vanished at higher densities as Trunec et al. ${ }^{10}$ found, but in this case at $10^{11} \mathrm{~cm}^{-3}$. Hutchinson and Patacchini ${ }^{16}$ used a PIC code to compute the rise in ion current from the OML value to the ABR value as collisions increased and compared their results with those of Lampe et al. and others. They also gave a scaling law for their curves. Nonetheless, these results for spheres are not useful for 
experimentalists because of the difficulty in constructing small, refractory spheres with even smaller insulated, conducting supports.

Unfortunately, none of the work reviewed above after the classic OML, ABR, and BRL papers is useful to experimentalists because computations were necessarily done case by case. There have been no general $I-V$ characteristics that could parametrized so that they can be compared with measurements. The data presented here will show that the OML theory fortuitously works well in a range of density and collisionality that is relevant to many industrial applications.

\section{Experiment}

Measurements were carried out in an 8-tube distributed helicon source described elsewhere ${ }^{17}$ and diagrammed in Fig. 1. Each tube is $5 \mathrm{~cm}$ in diam and $5 \mathrm{~cm}$ high, powered by a 3-turn loop antenna driven to 300 or $400 \mathrm{~W}$ at $13.56 \mathrm{MHz}$. The DC magnetic field is provided by annular NdFeB permanent magnets placed above the tubes. The gas is Ar at 15 mTorr. Probes are inserted into four ports at levels Z1 and Z2, 10.6 and $18.3 \mathrm{~cm}$, respectively, below the tubes. Two ports at X1 are directly below the tubes, and two at X2 are between the tubes, whose axes are separated by $17.8 \mathrm{~cm}$. The B-field at the probes is less than $10 \mathrm{G}$.
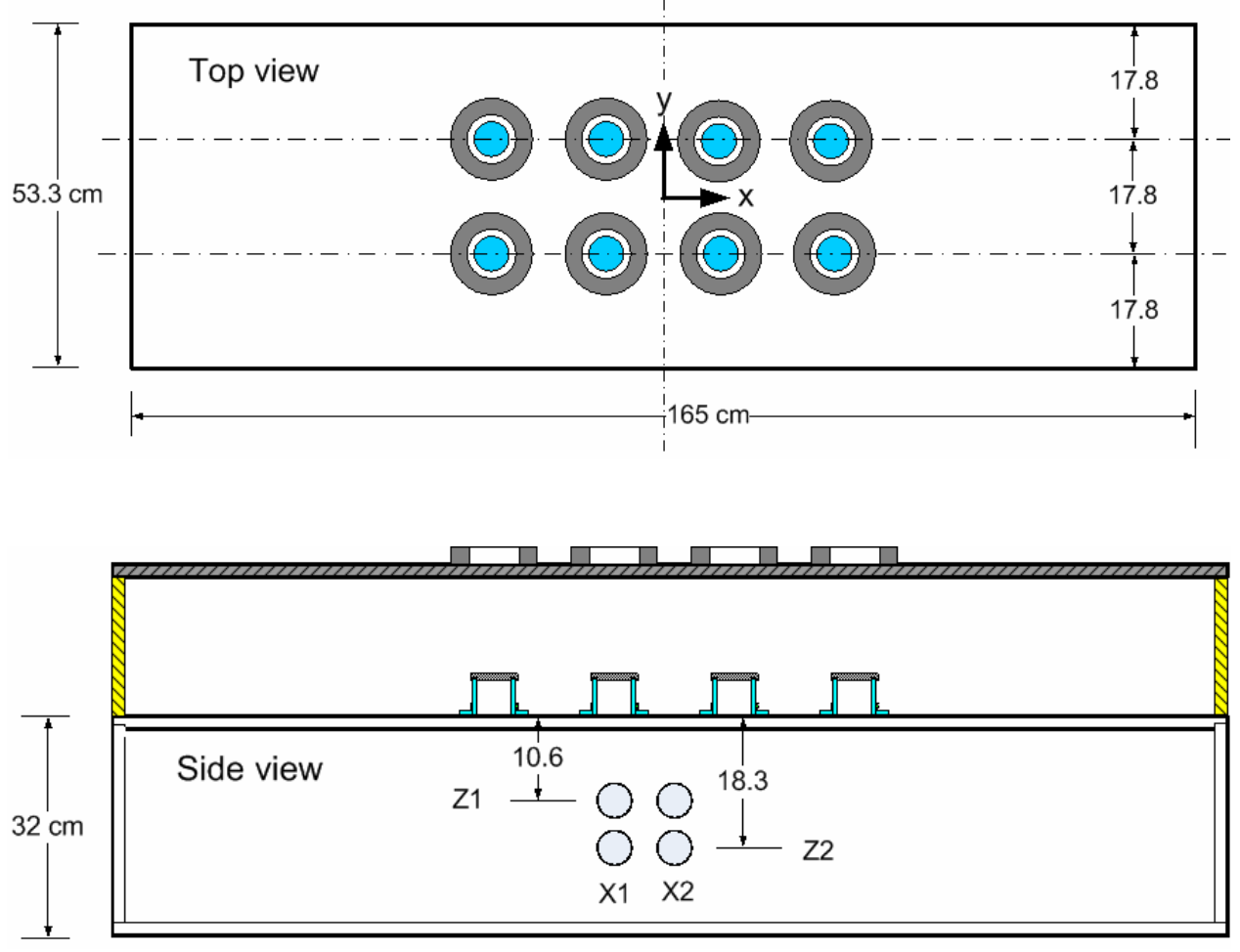

Fig. 1. Top and side views of the plasma source.

The probe tips were chosen thin and long to approximate cylinders with small $\xi_{\mathrm{p}}=R_{\mathrm{p}} / \lambda_{\mathrm{D}}$. Tungsten rods of 3-mil and 5-mil diam $\left(R_{\mathrm{p}}=38\right.$ and $\left.64 \mathrm{~nm}\right)$, and approximately $1 \mathrm{~cm}$ length $L$, were used. The aspect ratios $L / 2 R_{\mathrm{p}}$ were 132 and 78, respectively. Details of probe design and construction will be given in Sec. III. Probe $I-V$ characteristics were taken with a Hiden ESP Mk II electronics unit. This is an older model, but it has adjustable speed in the voltage sweeps. 
The importance of this will be shown in Sec. IV. Figure 2 shows a typical $I-V$ trace taken at $\mathrm{X} 2, \mathrm{Z} 2$ at $3200 \mathrm{~W}$ (400W per tube) with a 3-mil probe. This curve was analyzed as follows.

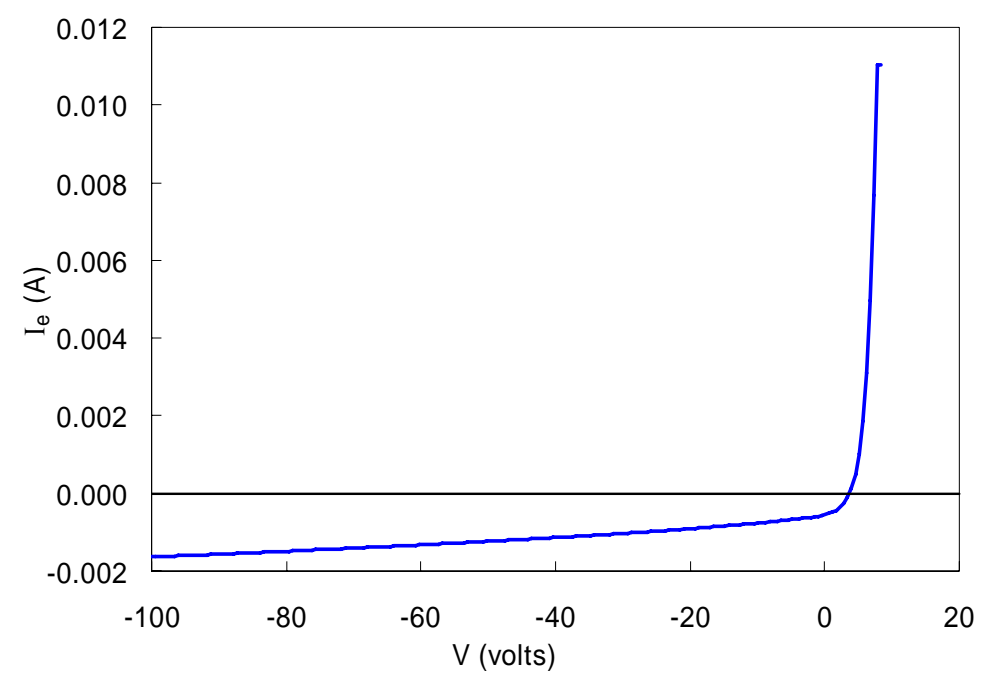

Fig. 2. A sample $I-V$ curve from an RF plasma.

Langmuir's OML theory ${ }^{18}$, as summarized by $\mathrm{Chen}^{1}$, gives the following approximate formula for saturation ion current $I_{\mathrm{i}}$ to a negatively biased cylindrical probe:

$$
I_{i}=A_{p} n e \frac{\sqrt{2}}{\pi}\left(\frac{e\left(V_{s 1}-V_{p}\right)}{M}\right)^{1 / 2}
$$

where $A_{\mathrm{p}}$ is the probe surface area, $e$ the electron charge, $V_{\mathrm{p}}$ the probe potential, and $M$ the ion mass. $V_{\mathrm{s} 1}$ is a temporary "space potential" used for fitting. Eq. (1) is an extremely useful formula, since it does not depend on a previous determination of $T_{\mathrm{e}}$; and $n$ can be determined knowing only the probe size and ion species. Eq. (1) predicts that a plot of $I_{\mathrm{i}}{ }^{2} v s . V_{\mathrm{p}}$ should be linear, as shown in Fig. 3. By adjusting $n$ and $V_{s 1}$, a least-squares fit of Eq. (1) can be made to the experimental points. It is seen that the $I^{2}-V_{\mathrm{p}}$ dependence is following almost exactly. The intercept at $V_{\mathrm{s} 1}$ is not the real space potential $V_{\mathrm{s}}$, since the OML curve should dip downwards a few $T_{\mathrm{i}}$ 's (or perhaps $T_{\mathrm{e}}$ 's) before reaching $V_{\mathrm{s}}$; but the fit is stopped well before this point. From this fit one obtains a density $n=4.78 \times 10^{11} \mathrm{~cm}^{-3}$. Argon ions are totally unmagnetized at $10 \mathrm{G}$.

Next, we assume that the bulk electron distribution is Maxwellian, so that

$$
I_{e}=A_{p} n e\left(K T_{e} / 2 \pi m\right)^{1 / 2} \exp \left[e\left(V_{p}-V_{s}\right) / K T_{e}\right]
$$

The ion current as given by the fitted line in Fig. 3 is subtracted from the raw current to obtain $I_{\mathrm{e}}$. A semilog plot of $I_{\mathrm{e}} v s . V_{\mathrm{p}}$ is shown in Fig. 4. Since $n$ is already known unambiguously from the OML ion current, the straight line fit in Fig. 4 gives $K T_{\mathrm{e}}$ from its slope and $V_{\mathrm{s}}$ from its horizontal position. Because of difficulties in measuring $I_{\mathrm{e}}$ correctly (Sec. IV), we do not determine $V_{\mathrm{s}}$ from the derivative of $I_{\mathrm{e}}$ to find $n_{\mathrm{e}}$ from that. As long as $I_{\mathrm{i}}$ follows OML scaling, the value of $n_{\mathrm{i}}$ from that is much more trustworthy than any value of $n_{\mathrm{e}}$ derived from the electron current. In Fig. 4, data are not taken beyond $I_{\mathrm{e}}=10 \mathrm{~mA}$ to avoid probe heating. Curves extending to $V_{\mathrm{s}}$ and beyond will be shown in Sec. IV. Here the value of $V_{\mathrm{s}}$ can be found without submitting the probe to large currents because $n$ is already known. The value of $K T_{\mathrm{e}}$ found by fitting between 
$V_{\mathrm{p}}=1$ and $8 \mathrm{~V}$ is $1.36 \mathrm{eV}$, and the corresponding value of $\xi_{\mathrm{p}}$ is 3.05 . Note that the straight part of $I_{\mathrm{e}}$ depends critically on subtraction of the correct amount of ion current, but the $T_{\mathrm{e}}$ value obtained is not changed if the data were extended further into the bulk distribution. There appears to be a "tail" of high energy electrons at the left of Fig. 4. Though the existence of such a tail is reasonable, it will be shown in Sec. IV that this tail is often spurious, though in some cases it may be real.

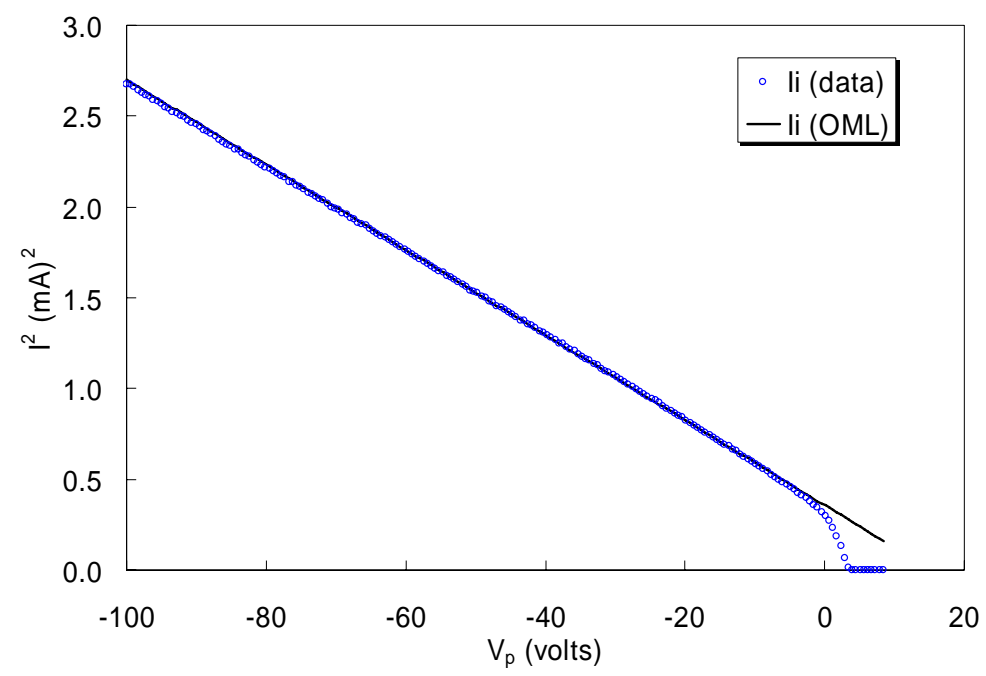

Fig. 3. Measured ion current squared (o) and an OML fit (-) from $-100 \mathrm{~V}$ to $-10 \mathrm{~V}$. Here $I^{2}$ is actually the total probe current $I_{\mathrm{p}}^{2}$, cut off at $V_{\mathrm{f}}$.

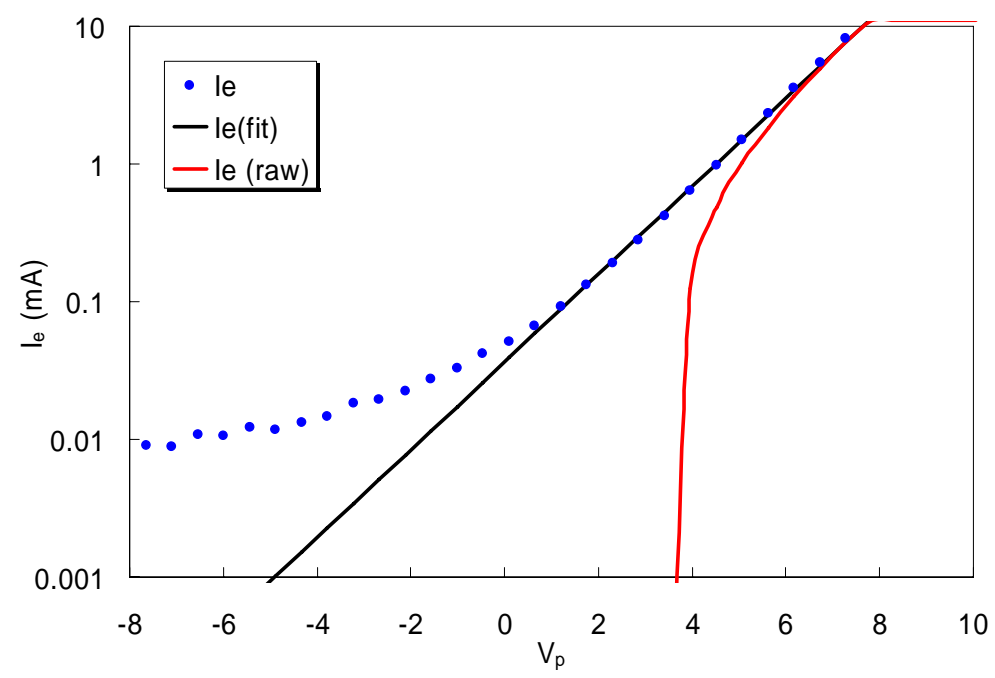

Fig. 4. Semilog plot of negative probe current $-I_{\mathrm{p}}$ vs. probe voltage before $(-)$ and after $(\bullet)$ subtraction of the ion current, as fitted by OML theory.

To see what other probe theories obtain with the same data, we have analyzed the $I-V$ curve of Fig. 2 using the ABR and BRL theories. The fitting was done using the parametrization of the ABR and BRL curves given by Chen ${ }^{1}$. Since $I_{\mathrm{i}}$ in these cases depends on $T_{\mathrm{e}}$, the fitting of $I_{\mathrm{i}}$ and $I_{\mathrm{e}}$ has to be done iteratively until the best fit is obtained for both curves. Figure 5 shows the result for the ABR case. Though it may not be obvious on this scale, the theoretical line is more curved than the data, and lies outside experimental error. The discrepancy is more noticeable with larger $R_{\mathrm{p}}$. The curvature near floating potential causes too little ion current to be added back to $I_{\mathrm{e}}$ to straighten out the $\ln I_{\mathrm{e}}$ curve, so that the part that is Maxwellian is shortened. 
Similar diagrams for the BRL case are shown in Fig. 6. Here again the theory predicts more curvature to $I_{\mathrm{i}}^{2}\left(V_{\mathrm{p}}\right)$ than is measured, and $\ln I_{\mathrm{e}}$ is straight only over a short range of $V_{\mathrm{p}}$. The dashed line on the BRL ion plot shows the form of a linear $I-V$ dependence. Clearly, the linear $I-V$ curves predicted $^{12}$ when collisions are important is not followed. The values of $n$ and $K T_{\mathrm{e}}$ derived from these theories are as follows:

$$
\begin{aligned}
& \text { OML: } n=4.78 \times 10^{11} \mathrm{~cm}^{-3}, K T_{\mathrm{e}}=1.36 \mathrm{eV} \\
& \text { ABR: } n=2.21 \times 10^{11} \mathrm{~cm}^{-3}, K T_{\mathrm{e}}=1.20 \mathrm{eV} \\
& \text { BRL: } n=6.26 \times 10^{11} \mathrm{~cm}^{-3}, K T_{\mathrm{e}}=1.24 \mathrm{eV}
\end{aligned}
$$

As usual, ABR yields a low density, while BRL gives a high a density. The OML value is reasonable in view of the RF power and the discharge brightness. Unfortunately, it was not possible to check the OML result against another diagnostic because the apparatus was designed for another purpose.
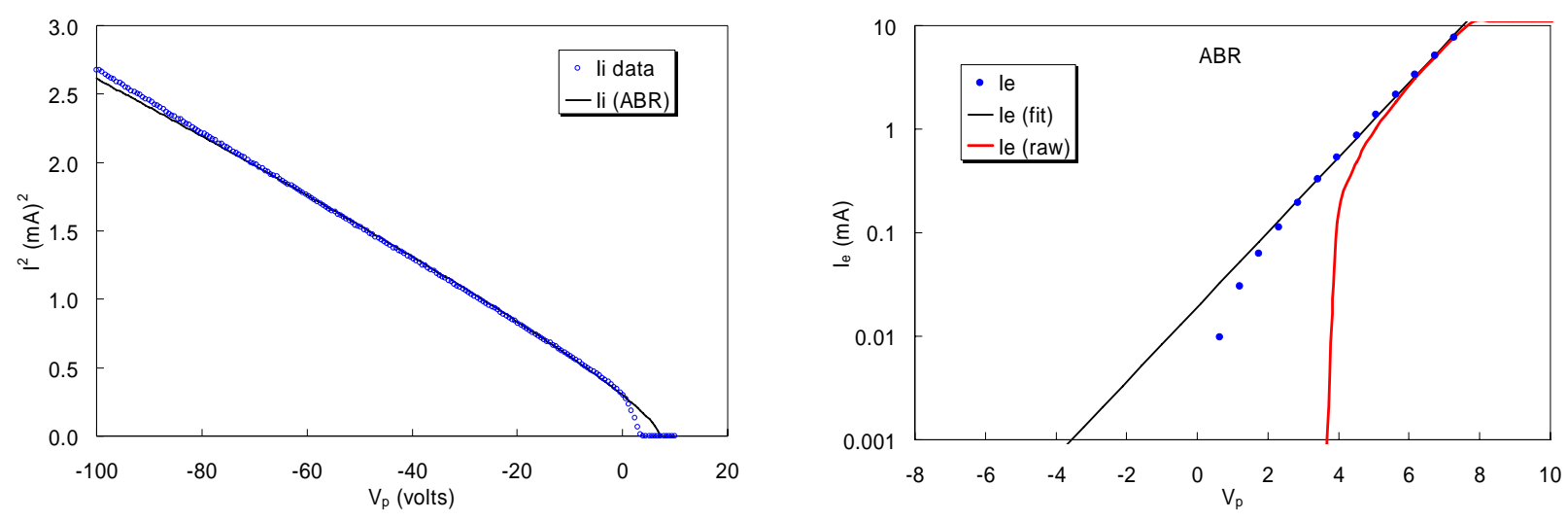

Fig. 5. Ion and electron fits using the ABR theory.
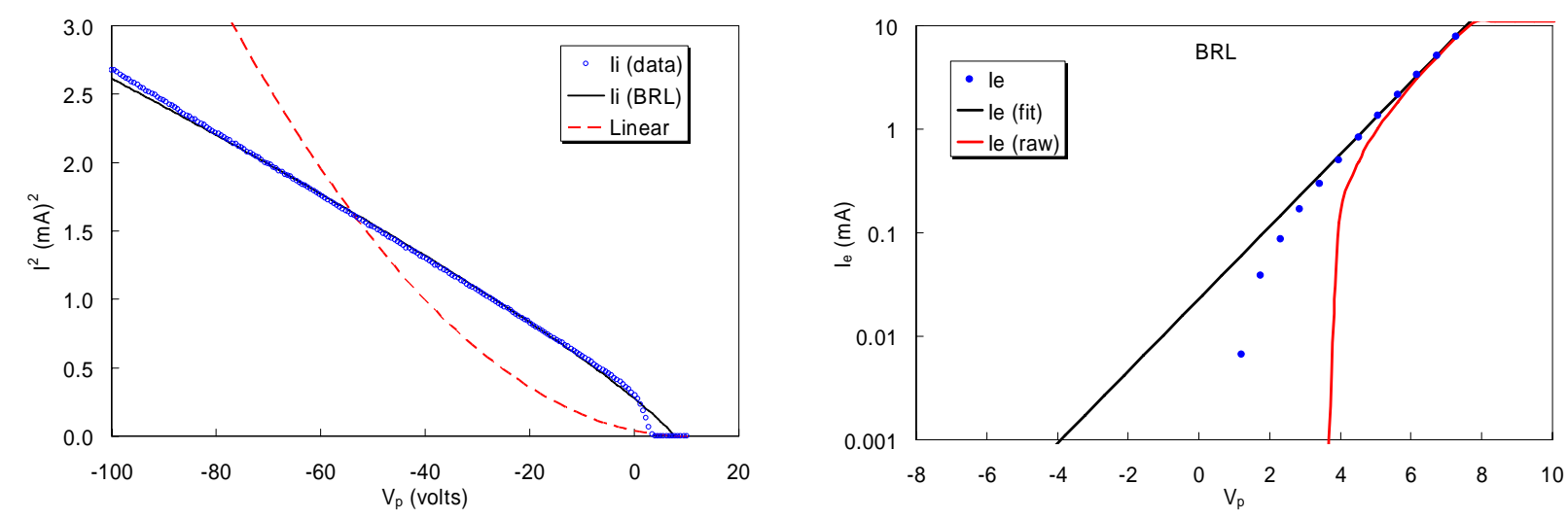

Fig. 6. Ion and electron fits using the BRL theory. The dashed line (-- $)$ is for $I_{\mathrm{i}} \propto\left(V_{\mathrm{s}}-V_{\mathrm{p}}\right)$.

\section{Probe construction}

In RF discharges, especially magnetized ones, it is essential to prevent fluctuations in $V_{\mathrm{s}}$ from distorting the $I-V$ characteristic. When $V_{\mathrm{s}}$ varies sinusoidally at the RF frequency, the probe current will vary non-sinusoidally because the $I-V$ curve is nonlinear. It is well known ${ }^{19,20}$ that simple averaging over the RF fluctuations in current will lead to spuriously high 
$T_{\mathrm{e}}$ 's and low $V_{\mathrm{f}}$ 's. A large number of schemes have been proposed historically to compensate for this effect, but the most effective has been to force the probe tip to follow the RF potential fluctuations so that $\left(V_{\mathrm{p}}-V_{\mathrm{s}}\right)$ remains at the value set by a DC power supply even if $V_{\mathrm{s}}$ is oscillating. This is done first by connecting the probe to the power supply through inductors (chokes) which have a large impedance at the RF frequency. Secondly, Sudit and Chen ${ }^{21}$ have found that this is insufficient, and a large, floating auxiliary compensation electrode has to be used to drive the probe tip to follow the $V_{\mathrm{f}}$ fluctuations, assuming that these are the same as the $V_{\mathrm{s}}$ fluctuations. Because even a short length of connecting wire has appreciable inductance and stray capacitance at RF frequencies, it is best to locate the compensation electrode and the choke(s) as close to the probe tip as possible. Several commercial probes have been designed along these lines. The probes used for this work are shown schematically in Fig. 7.

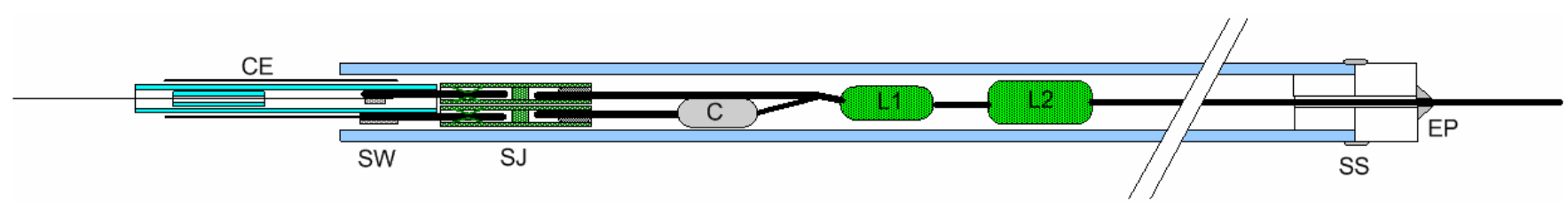

Fig. 7. Schematic of an RF-compensated probe.

The main shaft is a 1/4-in. $(6.4 \mathrm{~mm})$ diam ceramic $\left(\mathrm{Al}_{2} \mathrm{O}_{3}\right)$ tube made to be inserted into a vacuum mount with a single O-ring Wilson seal. Double seals could trap gas between the seals. The probe tips are 3-mil or 5-mil diam tungsten rods, which are the thinnest easily available. Thinner wires can be obtained, but they are curved. The tips are spot-welded (SW) to 30-mil tungsten rods using 1-mil Ni foil as flux. The rod is held by a spring-contact slip joint (SJ). The other end of the slip joint is soldered to the wire from choke L1. The probe length is defined by a small ceramic tube which actually covers the slip joint, though this overlap cannot be shown on the diagram. The small ceramic tube near the probe tip is used to center the tip and keep it away from any conducting deposits on the outside tube. The compensation electrode (CE) is a nickel foil wrapped around the probe ceramic tube and is connected by spot-welding (SW) to another 30-mil tungsten rod inserted into another slip joint. The back end of this joint is soldered to a small capacitor, which conducts the RF fluctuations to the probe lead at L1 but blocks the DC probe current. The vacuum seal is made at the back end of the probe shaft by a plastic plug machined to fit the ceramic tube and the probe wire. The wire is permanently sealed to the plug with epoxy (EP). The plug is sealed to the probe shaft with a soft-setting sealant (SS). The plug can thus be loosened and the entire probe assembly pulled out for repairs. Having the vacuum seal at the back end avoids expensive metal-to-ceramic seals at the probe end. However, the chokes are not air-cooled. The probe current in short sweeps will not usually overheat the chokes, whose resistance is about $12 \Omega$, but the probe shaft and its contents can become hot when left in a steady-state plasma. All solder joints are shielded from the plasma. A new probe tip is cleaned by ion bombardment at $-100 \mathrm{~V}$ one second at a time. It is then subjected to electron heating with great care; the probe melting condition is given in the next section.

Finding the right chokes for RF filtering requires great patience. The inductive impedance of small chokes is not large enough by itself; one has to use the large impedance at the self-resonance of the choke. The required impedance depends mainly on the impedance of the sheath on the probe tip and on the amplitude of the RF fluctuations in $V_{\mathrm{s}}$. The sheath impedance varies during the RF cycle in an irregular way. The problem has been treated in detail by $\mathrm{Chen}^{22}$. There it was found that accurate measurements of $T_{\mathrm{e}}$ would require, in a typical case, a choke impedance larger than $100 \mathrm{k} \Omega$ if $V_{\mathrm{s}}$ oscillations are as large as $100 \mathrm{~V}$. Our practice is to make probes with $Z_{\mathrm{c}} \geq 250 \mathrm{k} \Omega$ at the RF fundamental and somewhat smaller at the 
second harmonic. Since the resonance frequencies listed by manufacturers are actually minima, we have measured hundreds of chokes from many sources to find ones resonating at 13.56 and $27.12 \mathrm{MHz}$. Once found, chokes are usually connected in strings to increase the impedance; but this does not usually help. Fortunately, we have found a single commercial choke (L1) that has higher impedance than strings of chokes at $13.56 \mathrm{MHz}$. However, the resonance peak varies from sample to sample, so that the chokes have to be individually selected. The choke L2 does not have high impedance at $27.12 \mathrm{MHz}$, but broadly covers the high frequencies. The fluctuations at RF harmonics are expected to be small. The impedance vs. frequency characteristic of a typical selected choke pair is shown in Fig. 8. The $1 \mathrm{M} \Omega$ peak occurs below the RF frequency and shifts further down when the probe wire is connected, but $Z_{\mathrm{c}}$ is still $>500$ $\mathrm{k} \Omega$ at $13.56 \mathrm{MHz}$ and $\approx 200 \mathrm{k} \Omega$ at 27.12 .

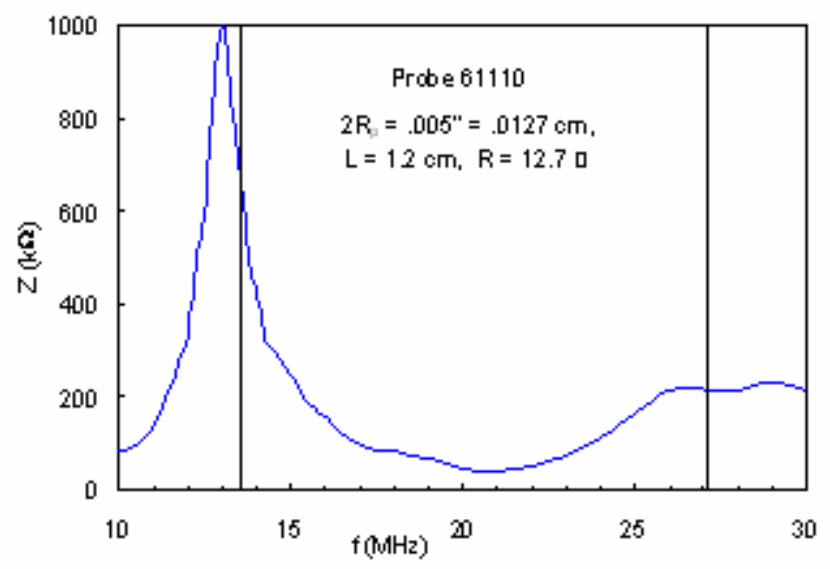

Fig. 8. Impedance characteristic of a sample probe. The vertical lines mark 23.56 and $27.12 \mathrm{MHz}$.

No expensive parts are needed for this probe, except perhaps for the alumina tubes. These are of standard dimensions, but the main tube has to be selected to have a thin enough wall to accommodate the L2 choke. If the probe is not exposed to CW plasma, glass tubes can be used; but can easily be broken carelessly. Accuracy of density determinations is limited to $\pm 5 \%$ by the error in measuring probe length if the tip is not perfectly straight, by current entering the sheath at the tip, and by creep of plasma under the ceramic tube.

\section{Experimental caveats}

1. Data accumulation rate. Probe curves are usually taken either by discharging a capacitor and recording the voltage and current as a function of time, or by using commercial digital electronics to change $V_{\mathrm{p}}$ step by step and recording $I_{\mathrm{p}}$ at each step. The sampling time at each step is limited at the low end by two factors a) the time it takes for a sheath to come to equilibrium, which is related to the ion plasma frequency, and b) the phase shift caused by the RF chokes, through which the $V_{\mathrm{p}}$ pulse has to pass. The ion plasma frequency at $n=10^{11} \mathrm{~cm}^{-3}$ is $10 \mathrm{MHz}$, so that dwell times longer than, say, $10 \mu \mathrm{sec}$ should be sufficient. However, much longer dwell times are necessary if there are low-frequency density oscillations such as from drift-wave type instabilities in the $10 \mathrm{kHz}$ range. To average over these requires dwell times longer than $1 \mathrm{msec}$. Some commercial units are designed to sweep an $I-V$ curve in much shorter times, and then to average over many sweeps. Though this could work in principle, we have previously experienced difficulties with such a procedure in an ECR (Electron Cyclotron Resonance) discharge in an $875 \mathrm{G}$ field. 
The Hiden ESP Mk II electronics unit used in this work permits adjusting the Start Dwell (the time $V_{\mathrm{p}}$ is applied before data taking), the Data Dwell (the time the probe current is averaged at each $V_{\mathrm{p}}$ ), the beginning and end $V_{\mathrm{p}}$ values, and the number of points in each sweep. In this study of the effect of dwell times, the notation $(\mathrm{s}, \mathrm{d})$ will be used to denote the Start and Data dwells in msec. First we measured $V_{\mathrm{p}}(t)$ applied to a $10 \mathrm{k} \Omega$ resistor at various $(\mathrm{s}, \mathrm{d})$. It was not possible to distinguish s and d; only the total dwell could be measured. Though dwells down to $1 \mathrm{msec}$ could be set, we found that dwells shorter than $3 \mathrm{msec}$ could not be produced. For dwells longer than about $5 \mathrm{msec}$, discrete steps of the proper length at the proper voltage were produced.

The first indication of trouble is shown in Fig. 9, in which $I-V$ curves of the same plasma near $V_{\mathrm{f}}$ are shown with different dwells and numbers of points per sweep. The curves are entirely different. Even if the dwells and the voltage range are kept constant, the curves differ when the number of points in a sweep is changed, as shown in Fig. 10. The electron distribution cannot be determined unambiguously. Contamination cannot be the cause because pure argon is used; however, thermionic emission could be the problem. The curves in Figs. 9 and 10 were swept from left to right. It turns out that the default setting leaves $V_{\mathrm{p}}$ at its final value for the duration of the default minimum cycle period of $1 \mathrm{sec}$, thus subjecting the probe to electron bombardment. A slow sweep, such as A in Fig. 9, allows the probe to cool off while the ion current is being collected.

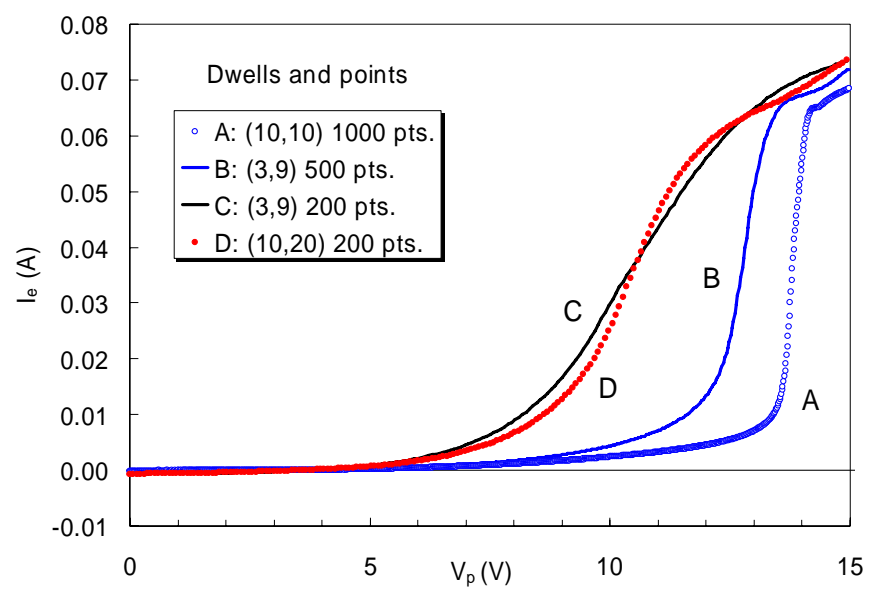

Fig. 9. Dwell test with a 5-mil probe at X1, Z2, y =0 in 15 mTorr of Ar at 2400W.

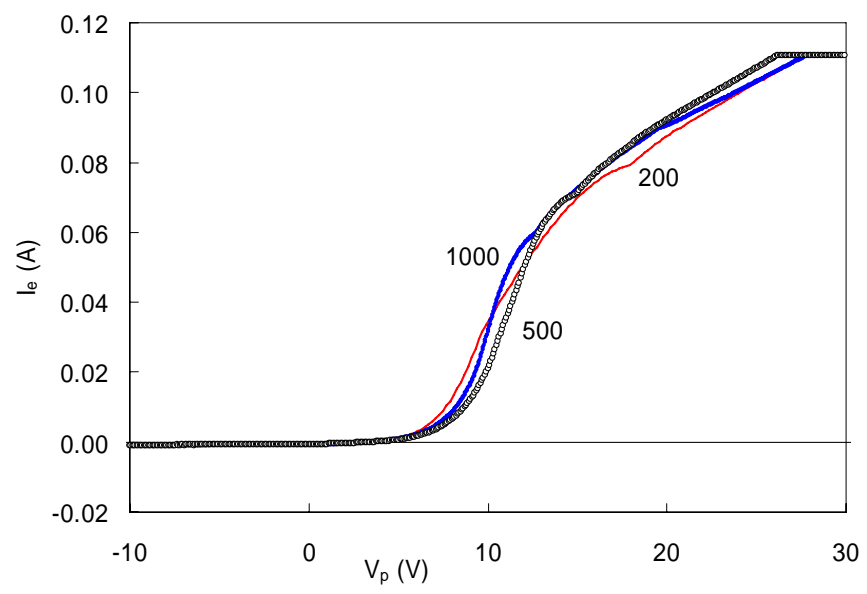

Fig. 10. Dwell test varying only the number of points in a sweep. 
The system was then reset to return the probe to $V_{\mathrm{f}}$ after a sweep, with the minimum cycle time as short as possible, and a second dwell test was performed with a 3-mil probe. Results are shown in Fig. 11. Variation with data dwell for the same sweep range and start dwell is shown for the electron part in (a) and the ion part in (b). Only the electron saturation current shows a small variation. The ion current is quite constant for all dwells, except that the $2-\mathrm{msec}$ setting causes fluctuations. In Fig. 11 (c) and (d), the dwells are kept at $(3,9)$ and the number of points in a scan is varied. The electron part in (c) shows variation, since the sweep extends to $+40 \mathrm{~V}$, and probe heating could be a factor. The ion part in (d) is limited to a maximum voltage of $+10 \mathrm{~V}$, and the ion current is reproducible for any number of points in the scan. Both ion and electron currents are reproducible if the step size, volts per point, is kept constant as the total voltage range swept is varied.

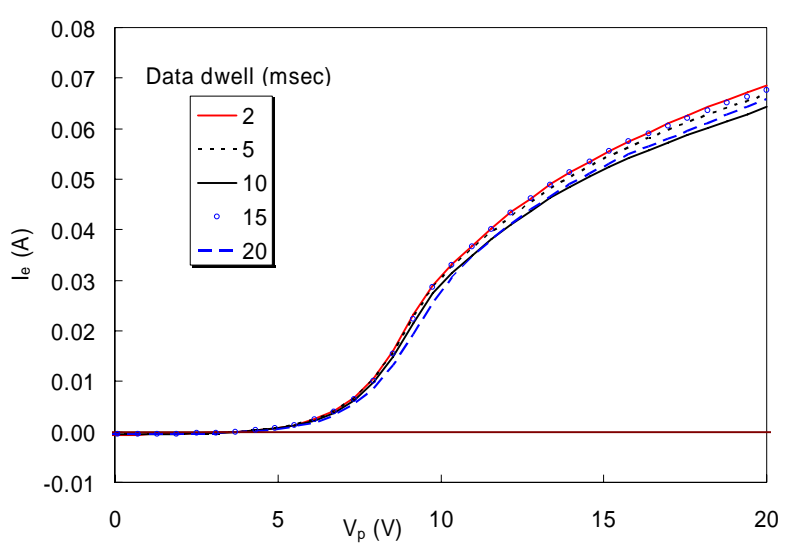

(a)

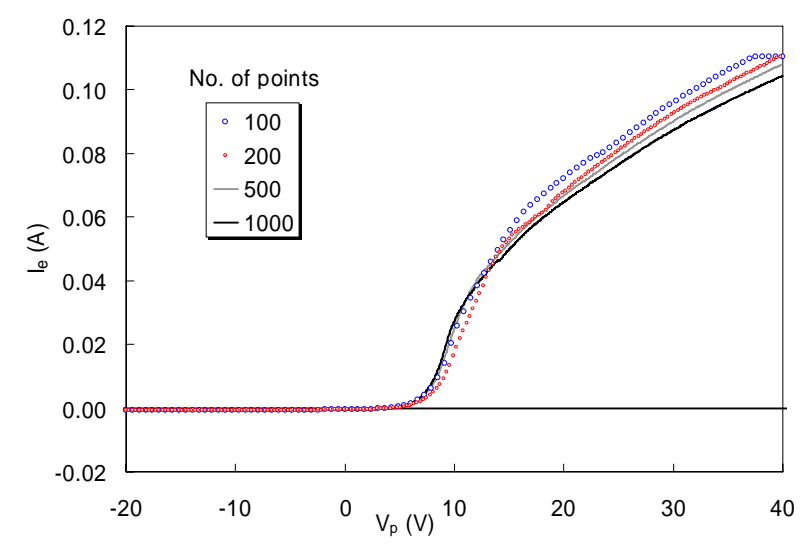

(c)

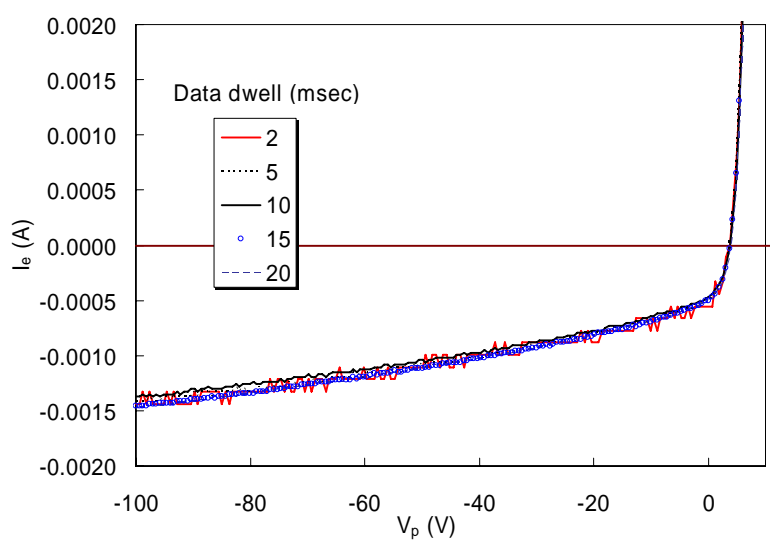

(b)

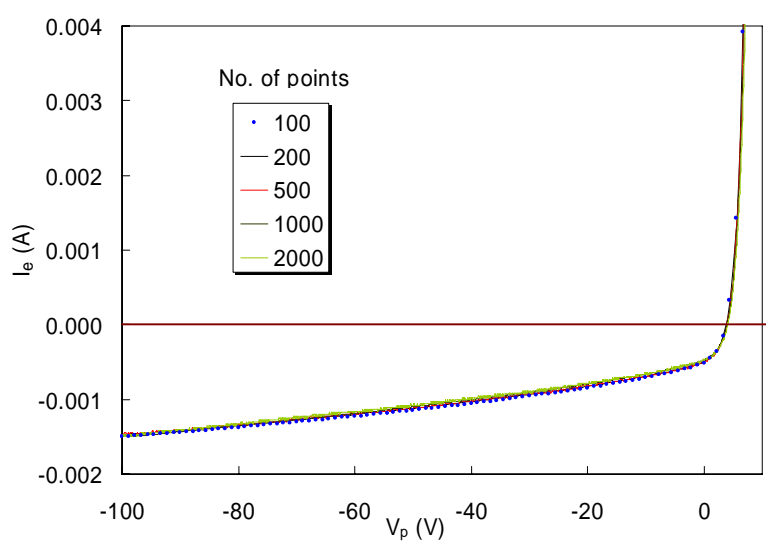

(d)

Fig. 11. Dwell test with a 3-mil probe at X2, Z2, $y=0$ in 15 mTorr of Ar at $2400 \mathrm{~W}$ : (a) $-100 \mathrm{~V}$ to $+20 \mathrm{~V}, 200$ points, start dwell 3 msec, electron part; (b) same, ion part; (c) $-20 \mathrm{~V}$ to $+40 \mathrm{~V}$, dwells $(3,9)$; (d) same, $-100 \mathrm{~V}$ to $+10 \mathrm{~V}$.

The conclusion from this study is that ion current can be measured accurately regardless of the data acquisition settings, but electron currents can vary. Double differentiations of $I_{\mathrm{e}}$ to get the electron energy distribution cannot be trusted. This result differs from that of Jauberteau $^{23}$, applying to much higher neutral pressures, that density is correctly given by electron current but not by ion current.

2. Probe heating. Tungsten melts at a black-body temperature of $3683 \mathrm{~K}$, but it starts thermionic emission at the rate of $1 \mathrm{~mA} / \mathrm{cm}^{2}$ at $2000 \mathrm{~K}$. If a thin probe tip is subjected to high 
positive potentials, the electron current can be large enough to bring the tip into emission. If a positive probe bias is applied for many milliseconds, the probe tip can come to a steady-state temperature given by the Stefan-Boltzmann law. A 3-mil diam tip will reach $2000 \mathrm{~K}$ with $I_{\mathrm{e}}=87$ $\mathrm{mA}$ at $V_{\mathrm{p}}=25 \mathrm{~V}, 43 \mathrm{~mA}$ at $50 \mathrm{~V}$, and $22 \mathrm{~mA}$ at $100 \mathrm{~V}$. The corresponding numbers for a 5 -mil tip are $145 \mathrm{~mA}$ at $25 \mathrm{~V}, 72 \mathrm{~mA}$ at $50 \mathrm{~V}$, and $36 \mathrm{~mA}$ at $100 \mathrm{~V}$. Figure 12 shows non-reproducibility of electron currents when voltage sweeps are made forward and backward between different ranges. The large currents in Fig. 12b are certainly due to electron emission, since the sweep was show due to the large dwell times and the large number of points. Furthermore, the probe remained at the final voltage after each sweep. Three factors govern the accuracy of $I_{\mathrm{e}}$ curves: 1) the largest $V_{\mathrm{p}}$ and how long it is applied, 2) the speed of a sweep, as determined by the dwell times and the number of points, and (3) the cooling time between sweeps. With a forward sweep starting at large negative $V_{\mathrm{p}}$, the probe has time to cool off while $I_{\mathrm{i}}$ is taken.

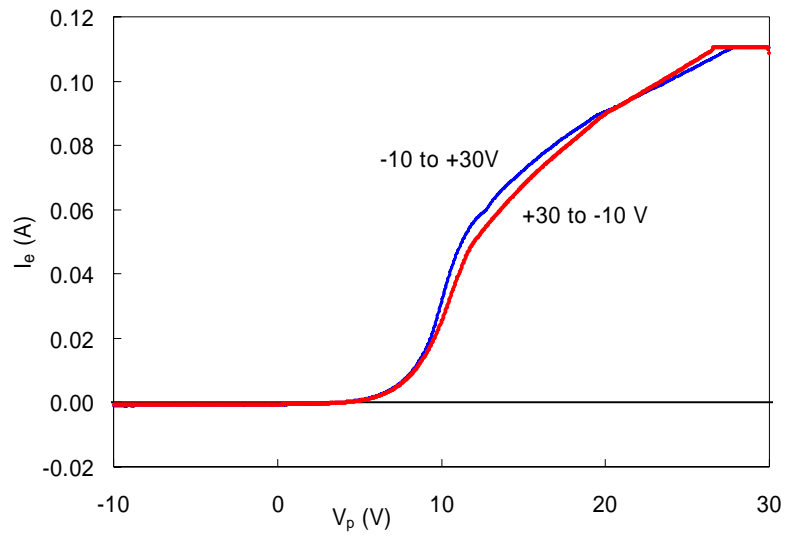

(a)

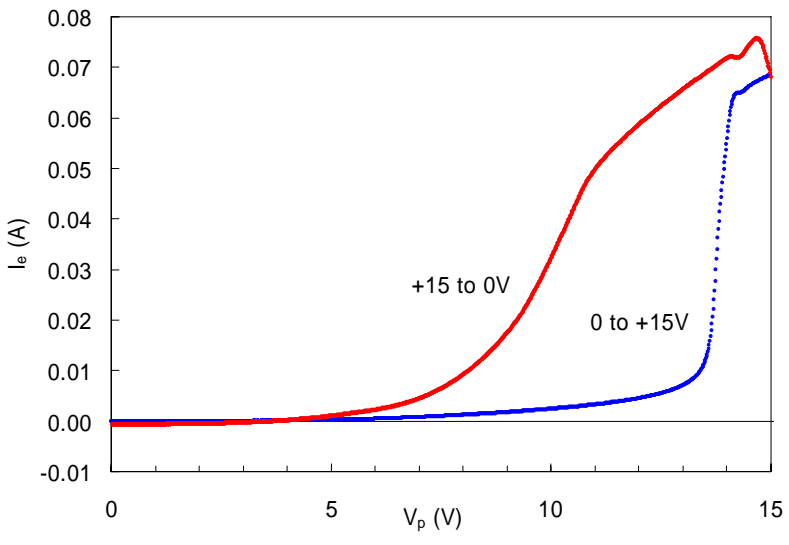

(b)

Fig. 12. $I-V$ curves taken forward and backward with a 5-mil probe at X1, Z2, $y=0,15$ mTorr, 2400W, 1000 points, dwells $(3,9)$ in (a) and $(10,10)$ in (b).

In principle, a probe can be heated to space-charge-limited emission by applying a high $V_{\mathrm{p}}$ and then quickly brought to negative $V_{\mathrm{p}}$ to act as a "hot probe". The floating potential would then be an accurate measurement of $V_{\mathrm{s}}$. However, there is a thin line between space-chargelimited emission and probe melting.

3. False detection of beams. After electron current is corrected by subtracting the ion current fitted by OML theory, one often finds evidence of fast electrons ("beams"), as seen in Fig. 4. A similar discharge is analyzed in Fig. 13. With large distortions from a Maxwellian, the electron distribution can be derived from the second derivative of $I_{\mathrm{e}}\left(V_{\mathrm{P}}\right)$. Here the beam current is $<1 \%$ of $I_{\mathrm{e}}$, and the data are not accurate enough for this. However, one can assume that the beam is a drifted Maxwellian with a density $n_{\mathrm{b}}$ and a temperature $T_{\mathrm{b}}$, centered at energy $E_{\mathrm{b}}$. The current from such a beam can be calculated straightforwardly with the result

$$
I_{b}=\frac{I_{0}}{2}\left\{e^{-x_{c}^{2}}+\left(\frac{\pi E_{b}}{K T_{b}}\right)^{1 / 2}\left[1 \mp \operatorname{erf}\left(\left|x_{c}\right|\right)\right]\right\}, \begin{aligned}
& x_{c}>0 \\
& x_{C}<0
\end{aligned}
$$

where

$$
I_{0}=e n_{b} A_{p}\left(\frac{2 K T_{b}}{\pi m}\right)^{1 / 2} \text {, and } x_{c}=\left(\frac{-e V_{p}}{K T_{b}}\right)^{1 / 2}-\left(\frac{E_{b}}{K T_{b}}\right)^{1 / 2} \text {. }
$$


Fitting the beam part in Fig. 13a with this formula gives the beam current given by the open circles. Adding this to the bulk Maxwellian, we obtain a 5-parameter bi-Maxwellian which can be fitted to the entire $I_{\mathrm{e}}$ curve. The resulting numbers are $K T_{\mathrm{e}}=1.32 \mathrm{eV}, V_{\mathrm{s}}=9.2 \mathrm{~V}, n_{\mathrm{b}}=3.9 \times$ $10^{7} \mathrm{~cm}^{-3}, K T_{\mathrm{b}}=3.68 \mathrm{eV}$, and $E_{\mathrm{b}}=10.3 \mathrm{eV}$; while the OML ion fit yields $n=2.95 \times 10^{11} \mathrm{~cm}^{-3}$.

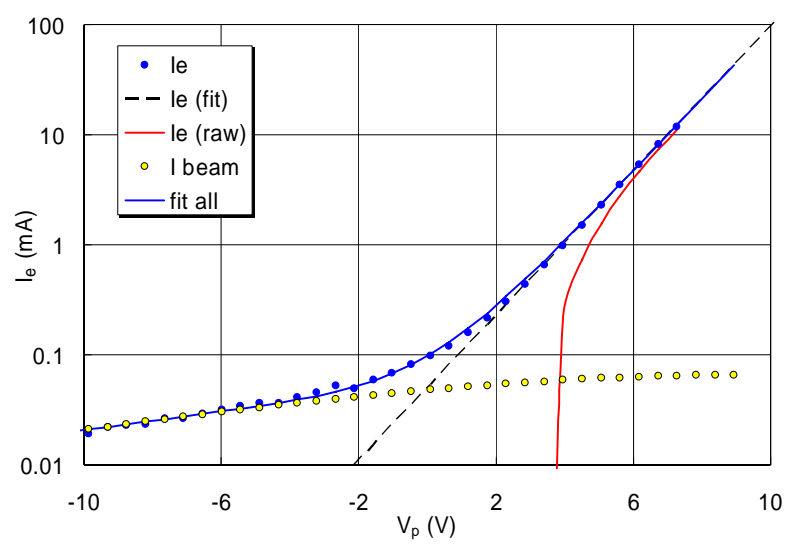

(a)

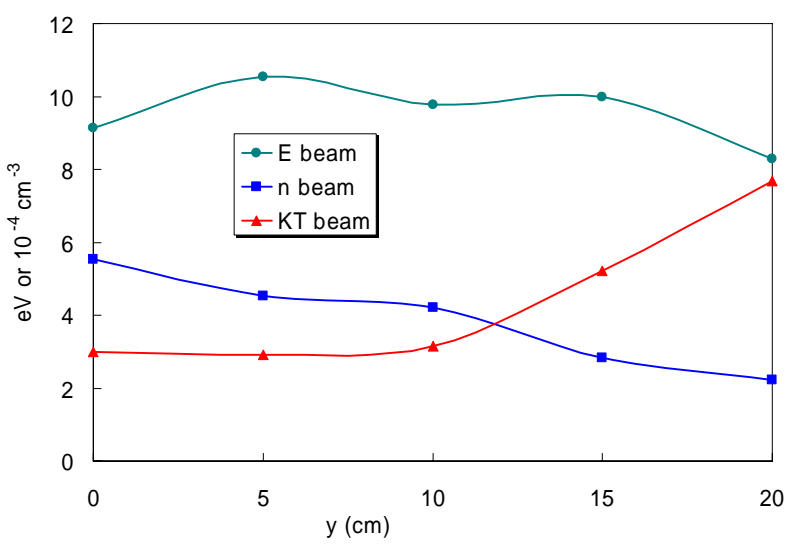

(b)

Fig. 13. (a) Semilog plot of $-I_{p}$ vs. $V_{\mathrm{p}}$ before (-) and after $(\bullet)$ subtraction of the OML ion current. The base Maxwellian is fitted with the dashed line (- - ), while the high-energy tail is fitted with a drifted Maxwellian (0). The blue curve (-) through the data points is the sum of the two Maxwellians. Data taken with a 5-mil probe at $\mathrm{X} 1, \mathrm{Z} 2, \mathrm{y}=0$ in $15 \mathrm{mTorr}$ of Ar at 2400W. (b) Variation of beam parameters with position. The midplane of the discharge is at $y=0$, and the near wall at $y=25 \mathrm{~cm}$.

The beam density is only $0.01 \%$ of $n$, and its presence is revealed deeply in the part of $I_{\mathrm{e}}$ that depends on the accuracy of the ion subtraction. Nonetheless, the numbers are of reasonable magnitude. Fast electrons caused by Landau damping in helicon discharges have been observed by Molvik et al. ${ }^{24}$ to the extent of $20 \mathrm{~mA} / \mathrm{cm}^{2}$ in a $5-\mathrm{kW}$ plasma with $n \approx 3 \times 10^{11} \mathrm{~cm}^{-3}$. Our probe area of $0.056 \mathrm{~cm}^{2}$ would collect $\approx 1 \mathrm{~mA}$, compared with the observed $0.1 \mathrm{~mA}$. However, there are two good reasons why the beam current in Fig. 13 should be $<<1 \mathrm{~mA}$. First, our power was only $300 \mathrm{~W}$ per tube, compared with $5 \mathrm{~kW}$. Second, our RF compensation method is incapable of detecting a beam phased with the RF, as shown by Chen ${ }^{25}$. The method depends on detecting the $V_{\mathrm{f}}$ oscillations and modulating the probe bias to follow them. However, a phased beam also modulates $V_{\mathrm{f}}$, so that compensated $I-V$ curve cannot see it. Only the DC part of a beam can be detected.

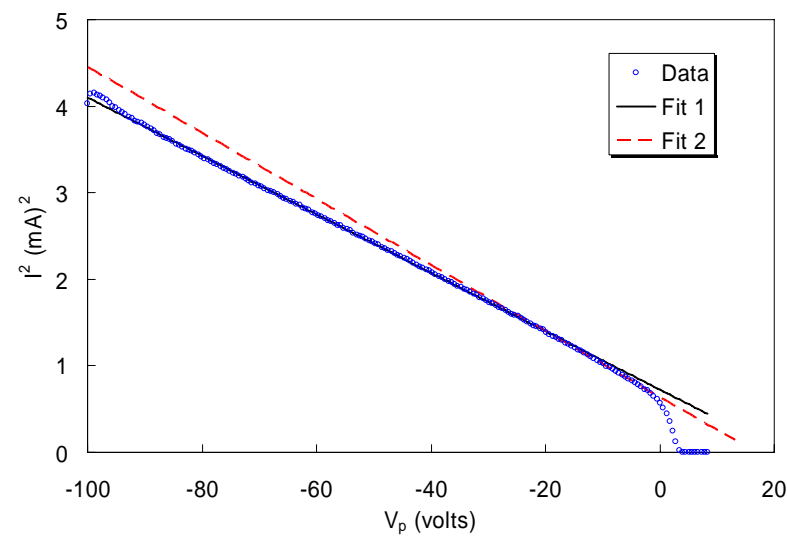

(a)

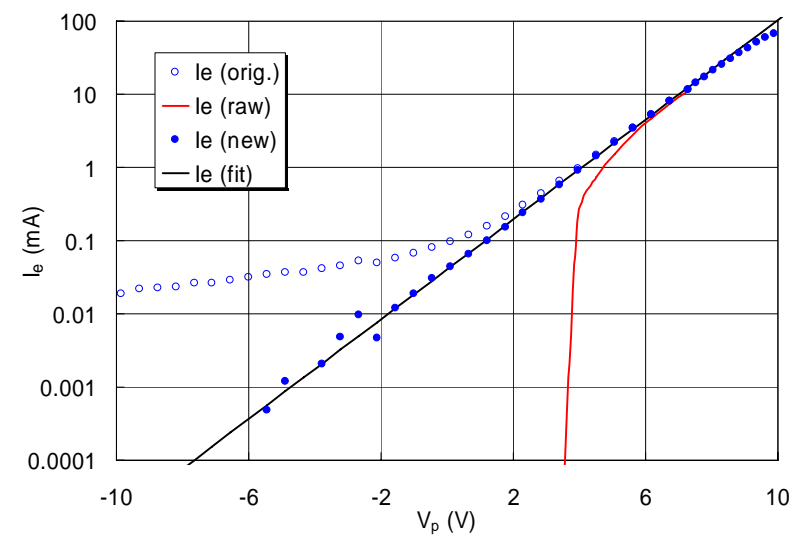

(b)

Fig. 14. (a) Fits to the ion data over different ranges. (b) Electron current as corrected with ion Fit 2. 
The derived values of $n_{\mathrm{b}}, T_{\mathrm{b}}$, and $E_{\mathrm{b}}$ are all reasonable for a helicon discharge. Furthermore, the "beam" seems to vary in a reasonable way with position, as shown in Fig. 13b. The fast electrons will thermalize as they diffuse toward the wall; hence, $n_{\mathrm{b}}$ is expected to fall and $T_{\mathrm{b}}$ to increase, as seen in Fig. 13b. $E_{\mathrm{b}}$ is more constant. The $n$ and $T_{\mathrm{e}}$ values of the bulk plasma vary in a similar direction, but more slowly. It would be tempting to conclude that OML theory is followed so closely in this experiment that beam fractions of order $10^{-4}$ can be detected, but this is deceptive. The $I_{\mathrm{i}}$ data often have a slight curvature that cannot easily be noticed, but it leads to a slightly different extrapolation. In Fig. 14a, the data have been fitted from $V_{\mathrm{p}}=-90 \mathrm{~V}$ to $-5 \mathrm{~V}$ (solid line), and from $-20 \mathrm{~V}$ to $-5 \mathrm{~V}$ (dashed line). If the second fit is used to correct the electron data, the "beam" disappears as seen in Fig. 14b. The electron distribution is then found to be Maxwellian over almost five orders of magnitude. The variation of $n_{\mathrm{b}}$ with position in Fig. $13 \mathrm{~b}$ may be caused by the change in the curvature of the ion plot at different densities. As the probe approaches the wall, the plasma density gets lower, the value of $\xi_{\mathrm{p}}$ decreases, and the OML theory is followed more closely. This reduces the curvature of the ion plot and makes the ion subtraction more accurate. Pilling and Carnegie ${ }^{26}$ have also found fast electron distributions which depend on the accuracy of ion subtraction, and they conclude that the beams are real. In our experiment, the beams are spurious in most cases; but sometimes the effect persists, and we cannot exclude the possibility that there are a few hyperthermal electrons.

4. Potential pulling. Many processing chambers are insulated so that the plasma has no connection to ground except through the probe electronics. When $I_{\mathrm{e}}$ to the probe is larger than the ion current to the walls, $V_{\mathrm{s}}$ can rise with $V_{\mathrm{p}}$. The electron saturation characteristic would then be linear, as shown in Fig. $15^{27}$. That this is caused by a change in $V_{\mathrm{s}}$ can be shown by measuring $V_{\mathrm{s}}$ changes with a floating probe nearby. $V_{\mathrm{s}}$ can be held constant by introducing a ground plane, near the probe, but diffusion to the ground plane would lower the density. It is better to introduce a second auxiliary electrode near the probe tip which measures the change in $V_{\mathrm{f}}$ with $V_{\mathrm{p}}$ with RF filtering. The change in $V_{\mathrm{s}}$ occurs on a timescale related to the establishment of a new equilibrium $n, T_{\mathrm{e}}$ profile in the discharge. Since this involves the motion of ions to the wall, it takes milliseconds. To avoid the change would require that $I_{\mathrm{e}}$ be drawn for $<<1 \mathrm{msec}$, which may not be consistent with the other time constraints discussed above. This effect does not happen in the present experiment because the discharge and the chamber are well grounded.

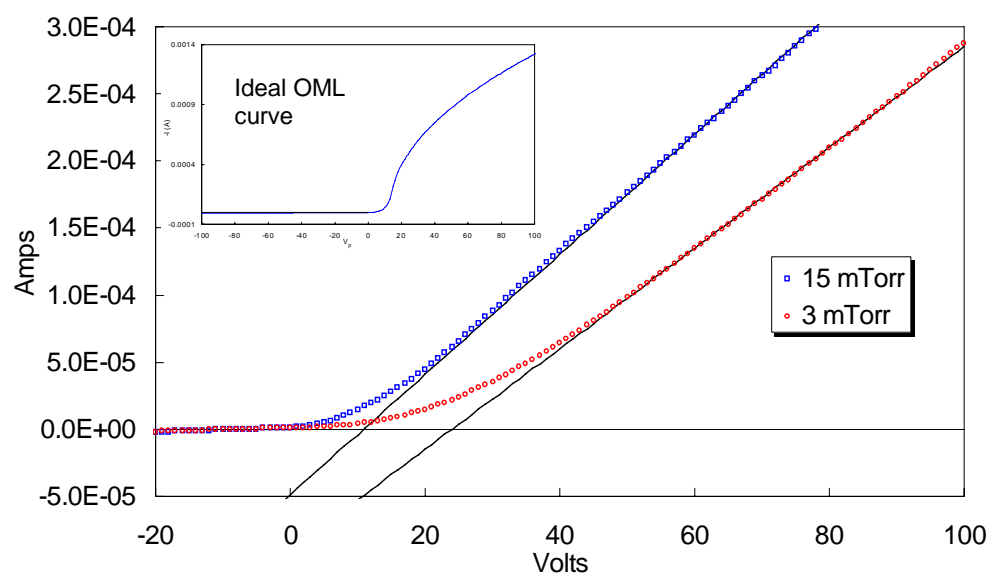

Fig 15. Example of potential pulling by saturation electron current.

V. Validity of OML theory. The OML theory of Mott-Smith and Langmuir ${ }^{18}$ assumes that ions with temperature $T_{\mathrm{i}}$ start from a sheath edge at $r=s$ far from the probe, and that the 
plasma potential $V_{\mathrm{s}}(r)$ varies so slowly that there is no absorption radius; that is, no effective black hole inside which the electric field is so strong that all ions reaching this radius are drawn into the probe. The theory neglects collisions. We now know that this is not realistic because collisions and ionization have to be considered. There is a non-ideal presheath which accelerates ions to energy $1 / 2 K T_{\mathrm{e}}$ at $r=s$, and the ion velocities are unidirectional. How, then, does the observed $I^{2}-V$ dependence arise? That dependence is indicative of ideal orbiting from infinity. If the ions were isotropic and all had energy $E_{\mathrm{i}}$, conservation of energy and angular momentum is sufficient to show that the ion current is proportional to $\left[1+\left(\mathrm{e}\left|V_{\mathrm{p}}\right| / E_{\mathrm{i}}\right)\right]^{1 / 2}$, so $I^{2} \propto V_{\mathrm{p}}$ if $e V_{\mathrm{p}}>>$ $E_{\mathrm{i}}$. If the ions are Maxwellian with temperature $T_{\mathrm{i}}$, the same relation obtains with $K T_{\mathrm{i}}$ replacing $E_{\mathrm{i}}$, aside from some numerical factors of order unity, if approximations are made. In this section, we show, first, that the exact OML theory does not give $I^{2} \propto V_{\mathrm{p}}$ unless some unreasonable assumptions are made. We then attempt to explain the $I^{2}-V$ dependence when there is a presheath; but the attempt is not entirely successful, and the experimental results are actually "surprising".

The simplicity of Eq. (1) is achieved only after several approximations, which we shall examine in more detail than done previously ${ }^{4}$. The ion current given by OML theory ${ }^{18,4}$ is

$$
I_{i}=A_{p} n e\left(K T_{i} / 2 \pi M\right)^{1 / 2} F,
$$

where $M$ is the ion mass and

$$
\begin{gathered}
F \equiv \frac{1}{\mathcal{E}} \operatorname{erf}\left(\Phi^{1 / 2}\right)+e^{\eta} \operatorname{erfc}(\Phi+\eta)^{1 / 2} \\
\eta \equiv-e V_{p} / K T_{i}>0 \\
\mathcal{E} \equiv R_{p} / s<1, \quad \Phi \equiv \eta /\left(\varepsilon^{-2}-1\right), \quad \Phi+\eta=\eta /\left(1-\mathcal{E}^{2}\right) .
\end{gathered}
$$

Here $\operatorname{erf}(x)$ is the error function and $\operatorname{erfc}(x)$ its complement. For simplicity $V_{\mathrm{s}}$ is set to 0 temporarily. We first assume $\varepsilon<<1$, the basic OML approximation. Then $\Phi^{1 / 2} \approx \varepsilon \eta^{1 / 2}$ and $(\Phi$ $+\eta) \approx \eta$. To proceed further, we must make the unreasonable assumption $\Phi^{1 / 2}<<1$. For small $x$, $\operatorname{erf}(x) \approx 2 x / \sqrt{ } \pi$, so that $\varepsilon$ cancels, and we have

$$
F(\eta) \approx \frac{2}{\sqrt{\pi}} \eta^{1 / 2}+e^{\eta} \operatorname{erfc}\left(\eta^{1 / 2}\right) .
$$

This same equation was derived without assumptions by Allen $^{28}$ by virtue of neglecting the sheath edge altogether, setting $s$ to infinity $a b$ initio so that $\varepsilon$ never appears in the theory. Yet here we had to assume $\varepsilon \eta^{1 / 2}<<1$ to obtain the same result. This is the same problem as the "cold-ion paradox" mentioned by Allen, and the resolution is subtle. Whether $\Phi$ is large or small depends on whether or not $\varepsilon \rightarrow 0$ faster than $T_{\mathrm{i}}^{1 / 2}$. Since $\eta$ is normalized to $T_{\mathrm{i}}, \eta^{1 / 2}$ is a large number, and $\varepsilon$ has to be extremely small for the approximation $\Phi^{1 / 2}<<1$ to hold. However, if one assumes $\varepsilon=0$ initially, that approximation is always valid. The same situation arose with the Bernstein-Rabinowitz theory ${ }^{5}$ vs. the Allen-Boyd-Reynolds theory ${ }^{3}$. Both theories calculated $V(r)$ starting from infinity, without the artificial introduction of a sheath edge. The BR theory assumed finite $T_{\mathrm{i}}$, while the ABR theory assumed $T_{\mathrm{i}}=0$, allowing no angular momentum in the problem. When the BR results were taken to the $T_{\mathrm{i}} \rightarrow 0$ limit, the result was different from that of ABR. The discrepancy was attributed by BR to a problem of nonuniform 
convergence. The physical situation with collisions, pre-sheaths, etc. did not enter into this purely mathematical problem. Since we do have a distinct sheath edge in practice (a place where the scalelength of $V(r)$ changes from the collision or ionization mean free path to the Debye length), Langmuir's postulate of a finite $s$ seems more reasonable than Allen's $s=\infty$ starting point. Unfortunately, Langmuir's theory needs an unjustifiable approximation to get the same result.

Regardless of how Eq. (9) was derived, a second approximation is needed to arrive at a linear $I^{2}-V$ curve. This is $\eta^{1 / 2}>>1$, which is a good approximation if $T_{\mathrm{i}}$ is near the gas temperature but not as good if $T_{\mathrm{i}} \approx T_{\mathrm{e}} / 2$ at the sheath edge. The asymptotic expression for $\operatorname{erf}(x)$ yields

$$
\operatorname{erfc}\left(\eta^{1 / 2}\right) \approx \frac{e^{-\eta}}{(\pi \eta)^{1 / 2}}\left(1-\frac{1}{2 \eta}+\frac{3}{4} \frac{1}{\eta^{2}}-\ldots\right)
$$

If we keep only the first term of this series, we obtain

$$
F(\eta) \approx \frac{2}{\sqrt{\pi}} \eta^{1 / 2}+e^{\eta} e^{-\eta} \frac{1}{(\pi \eta)^{1 / 2}}=\frac{1}{\sqrt{\pi}}\left(2 \eta^{1 / 2}+\eta^{-1 / 2}\right) \approx \frac{2}{\sqrt{\pi}} \eta^{1 / 2} .
$$

Using this in Eq. (5) gives

$$
I_{i}=A_{p} n e\left(\frac{K T_{i}}{2 \pi M}\right)^{1 / 2} \frac{2}{\sqrt{\pi}}\left(\frac{-e V_{p}}{K T_{i}}\right)^{1 / 2}=A_{p} n e \frac{\sqrt{2}}{\pi}\left(\frac{-e V_{p}}{M_{i}}\right)^{1 / 2},
$$

which is Eq. (1) when $V_{\mathrm{s}}$ is reinserted. The temperature, whether it is $T_{\mathrm{i}}$ or $T_{\mathrm{e}}$, has canceled out. This, finally, is the origin of the OML formula that predicts $I^{2} \propto V_{\mathrm{p}}$ with a coefficient that depends on neither $T_{\mathrm{i}}$ nor $T_{\mathrm{e}}$.

Just how bad are the approximations we have made? We next compare the exact formula of Eqs.(5) and (6) with the normally used approximate formula, Eq. (12). The following graphs are computed for a 5-mil probe $1 \mathrm{~cm}$ long in a plasma with $n=3 \times 10^{11} \mathrm{~cm}^{-3}$. The gas temperature $T_{\mathrm{n}}$ is estimated from the rise in neutral pressure when the discharge is on, going from 15 to $\approx 35$ mTorr. Neglecting the change in pumping rate, which varies only with $\sqrt{ } T_{\mathrm{n}}$ when the pump is throttled, the gas temperature should rise from $295 \mathrm{~K}$ to $688 \mathrm{~K}$. If the ions are in equilibrium with the gas, $K T_{\mathrm{i}}$ is $\approx 0.06 \mathrm{eV}$. Figure 16a shows the exact $I^{2}-V$ curves computed with this $T_{\mathrm{i}}$ for various values of $\varepsilon=R_{\mathrm{p}} / s$. The curve is linear only if $s$ is 500 times larger than $R_{\mathrm{p}}\left(R_{\mathrm{p}} / s<.002\right)$. For smaller sheaths, the lines curve into saturation as more orbits intersect the probe. The smallest $\varepsilon$ which whose curve is distinguishable from the line of the "simple" formula is 0.005 . Figure $16 \mathrm{~b}$ shows the curves for this $\varepsilon$ at various $T_{\mathrm{i}}$. For $T_{\mathrm{i}}$ between 0.5 and $1 \mathrm{eV}$, the curve is indistinguishable from the "simple" straight line. For larger $T_{\mathrm{i}}$, the curve is still straight but displaced upwards because the random ion current at $V_{\mathrm{p}}=V_{\mathrm{s}}$ is not negligible. In principle, this can be corrected for, but for reasonable $T_{\mathrm{i}}$ 's the correction is smaller than the experimental error. Thus, the "simple" formula, which is followed by experiment, does not agree with the exact OML theory unless $s$ is unreasonably large or $T_{\mathrm{i}}$ unreasonably high.

However, if we consider that at the sheath edge the ions have the Bohm velocity (a fact unknown to Langmuir), $T_{\mathrm{i}}$ could be much higher than the gas temperature. We therefore try next to rescue Langmuir's theory by varying the conditions at his sheath edge $s$. 


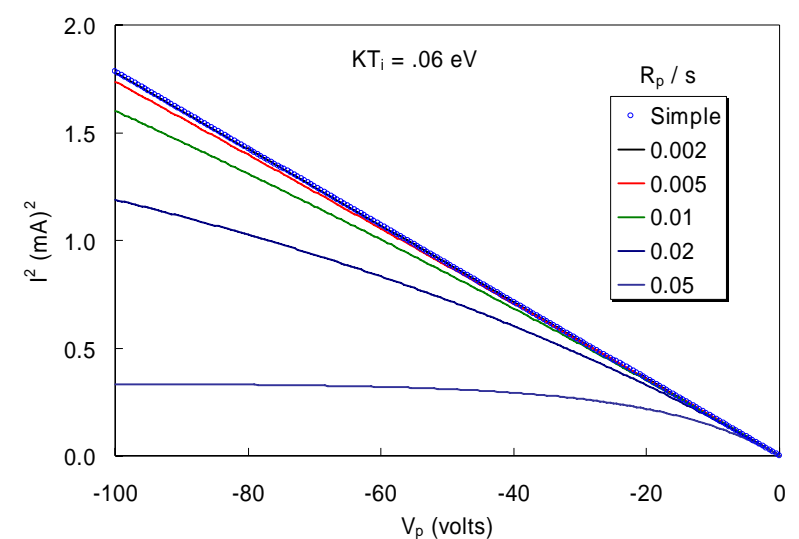

(a)

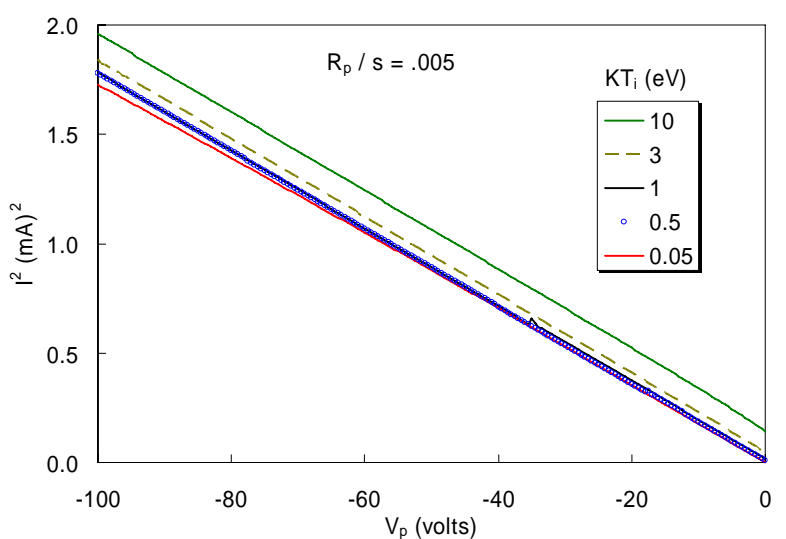

(b)

Fig. 16. (a) Exact OML curves for various $\varepsilon$ with $T_{\mathrm{i}}=T_{\mathrm{n}}$. The approximate OML formula is shown by the points (o). (b) Variation of exact OML curves with $T_{\mathrm{i}}$ for $\varepsilon=0.005$. In each case, the order of the curves follows that in the legend.

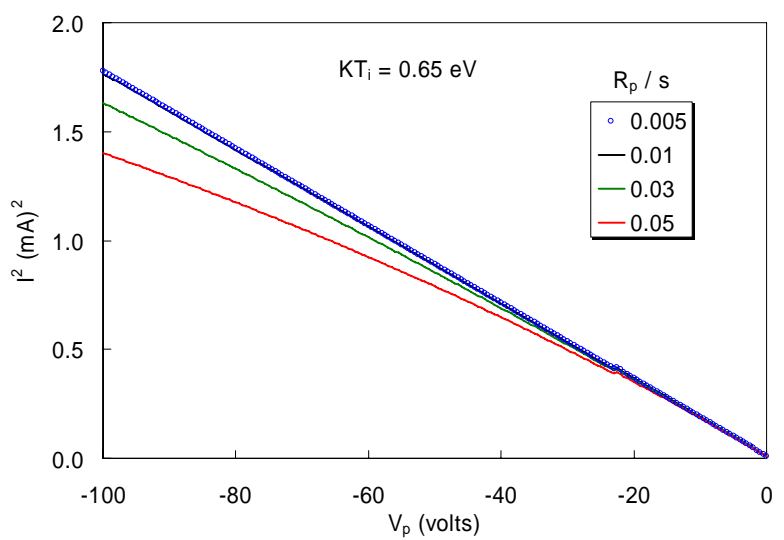

(a)

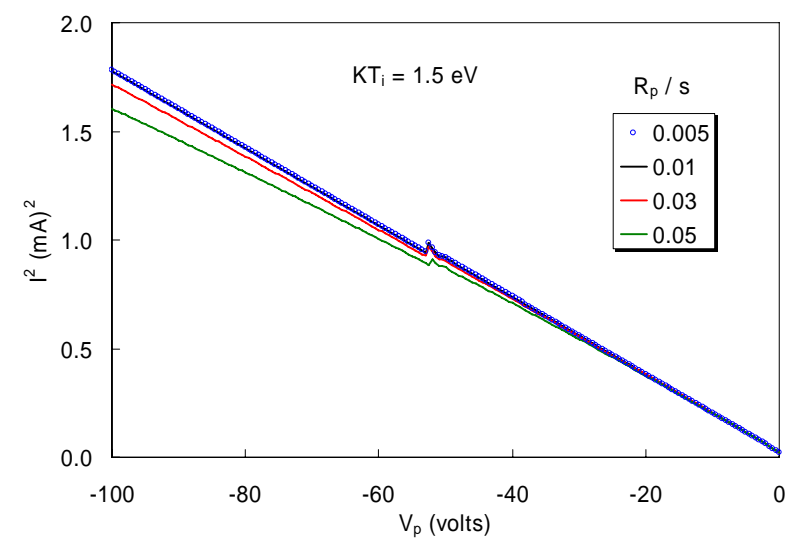

(b)

Fig. 17. Exact OML curves for various $\mathcal{E}$ with (a) $K T_{\mathrm{i}}=0.65 \mathrm{eV}$; (b) $K T_{\mathrm{i}}=1.5 \mathrm{eV}$. The kink in the curves is caused by switching to the asymptotic formula for $\operatorname{erfc}(x)$ when $e^{\eta}$ is large enough to cause numerical overflow.

At high $T_{\mathrm{i}}$, the sheath field is not strong enough to pull in many orbiting ions. Since ions at the sheath edge actually have an energy corresponding to $1 / 2 T_{\mathrm{e}}$, in Fig. 17 we have calculated the curves for $K T_{\mathrm{i}}=0.65 \mathrm{eV}$, corresponding $K T_{\mathrm{e}} \approx 1.3 \mathrm{eV}$ in downstream plasma, and for $K T_{\mathrm{i}}=$ $1.5 \mathrm{eV}$, corresponding to $K T_{\mathrm{e}} \approx 3 \mathrm{eV}$ inside the discharge. We have placed Langmuir's sheath edge $s$ at the radius where the Bohm criterion $v_{\mathrm{i}}=c_{\mathrm{s}}$ is satisfied. Collisions in the presheath will give the ions a spread in energy and angle, modifying the Bohm criterion, but the situation is not close to the isotropic distribution that Langmuir assumed. This difference can be accommodated by shifting the position of the sheath edge. Fortunately, the curves in Fig. 17 are independent of $\varepsilon$ as long as it is $\leq 0.02$. For such sheath widths, linear $I^{2}-V$ curves can be obtained by applying the Bohm criterion.

We next consider whether $\varepsilon \leq 0.02$ is consistent with experiment. The radius $s$ at which $|e V|=1 / 2 K T_{\mathrm{e}}$ can be read from curves of $V(r)$ given by Laframboise ${ }^{6}$ for a cylindrical probe biased to $25 K T_{\mathrm{e}}$ for $\xi_{\mathrm{p}}=R_{\mathrm{p}} / \lambda_{\mathrm{D}}=1$ and 10 . These yield $\mathrm{s} / \lambda_{\mathrm{D}}=8$ and 18 , respectively, giving $\varepsilon=R_{\mathrm{p}} / \mathrm{s}=$ $\left(R_{\mathrm{p}} / \lambda_{\mathrm{D}}\right) /\left(\mathrm{s} / \lambda_{\mathrm{D}}\right)=0.13$ and 0.56 . One case using $\mathrm{ABR}$ theory was computed by Chen and Arnush $^{29}$, yielding $s / \lambda_{\mathrm{D}}=21$ at $\xi_{\mathrm{p}}=15$, or $\varepsilon=0.71$. The experimental values of $\xi_{\mathrm{p}}$ measured in our experiment are around 3, which is closest to the $\varepsilon=0.13$ result. This is much too large for the OML characteristic to be linear. The ion-neutral charge-exchange mean free path $\lambda_{\mathrm{m}}$ was 
around $2 \mathrm{~mm}$, compared with $s=R_{\mathrm{p}} / \mathcal{E} \approx 0.29 \mathrm{~mm}$, so that this thin a sheath would be collisionless. However, if Langmuir's $s$ is taken at $\lambda_{\mathrm{m}}, \varepsilon$ would be 0.02 , in the range where the $I^{2}$ $-V$ curve is linear if $T_{\mathrm{i}}=1.5 \mathrm{eV}$, but nonlinear if $T_{\mathrm{i}}=0.65 \mathrm{eV}$, as seen in Fig. 17. The problem is that $T_{\mathrm{i}}$ should be closer to $0.1 \mathrm{eV}$ this far from the probe, and Fig. 16 shows that the curve is far from linear at $\varepsilon=.02$ at this temperature. No amount of fudging can explain the extremely linear $I^{2}-V$ curves measured.

The success of the simple OML formula in matching experimental data is apparently fortuitous. First, the formula differs from the exact formula because of unjustified approximations, and second, the exact formula was derived without knowledge of ion acceleration in a presheath controlled by collisions and ionization.

Regardless of the linearity of $I^{2}-V$, the exact OML theory requires the absence of an absorption radius. The criterion for a sufficiently smoothly varying $V(r)$ quoted by $\mathrm{Chen}^{4}$ is as follows:

$$
|e V(r)|>g(r)\left|e V_{p}\right|-(1-g) E_{s},
$$

where

$$
g(r)=\frac{R_{p}^{2}}{r^{2}} \frac{s^{2}-r^{2}}{s^{2}-R_{p}^{2}},
$$

and $E_{\mathrm{s}}$ is the ion energy at the sheath edge $s$. This critical profile is plotted in Fig. 18 for a 3-mil probe, $E_{\mathrm{i}}=1 / 2 K T_{\mathrm{e}}=1.3 \mathrm{eV}$, and, $R_{\mathrm{p}} / \lambda_{\mathrm{D}}=10$, which corresponds to $\varepsilon=0.56$. This large value of $\varepsilon$ is chosen because there is no $V(r)$ to compare it to except the aforementioned curve by Laframboise, which includes orbiting. In this case the $V(r)$ profile indeed is less steep than the critical profile. The density under these conditions, however, turns out to be $4.9 \times 10^{12} \mathrm{~cm}^{-3}$. With the longer $\lambda_{\mathrm{D}}$ 's at $3 \times 10^{11} \mathrm{~cm}^{-3}$, both curves would extend farther, but their relative positions should not change. Thus, the potential profiles with complete orbiting satisfy the validity condition for OML theory, but the values of $s$ and $T_{\mathrm{i}}$ that lead to straight $I^{2}-V$ curves are difficult to fathom.

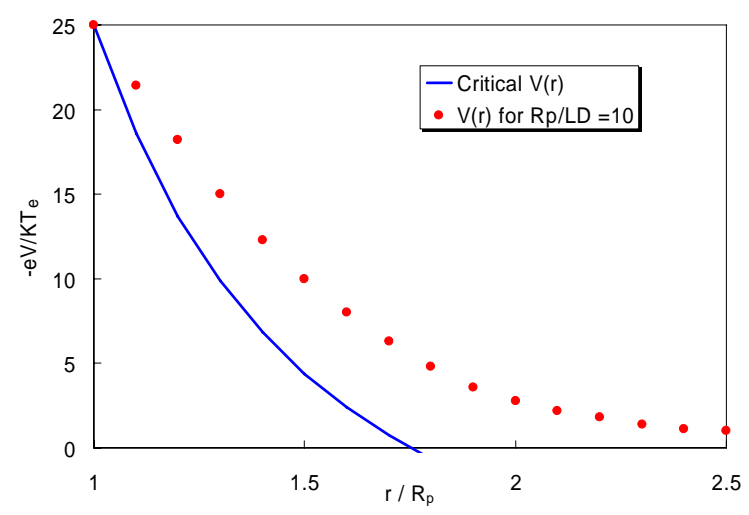

Fig. 18. Critical $V(r)$ for OML validity $(-)$ compared with a computed profile $(\bullet)$ for BRL theory

Being the hotter species, electrons do not suffer presheath acceleration, and their saturation currents can be computed from Eqs. (5) and (6) with a change of the sign of $\eta$ and changes from $T_{\mathrm{i}}$ to $T_{\mathrm{e}}$ and $M$ to $m$. Figure 19 shows a case in which $I_{\mathrm{e}}$ was measured successfully beyond $V_{\mathrm{s}}$ without emission problems. The fit with OML theory is very close, in spite of the fact that there was a magnetic field of order 10G, giving the electrons a Larmor radius of order 3-4 
mm. The space potential of $10 \mathrm{~V}$ obtained from the maximum of $\mathrm{d} I_{\mathrm{e}} / \mathrm{d} V$ agrees with that computed by the method in Sec. II.

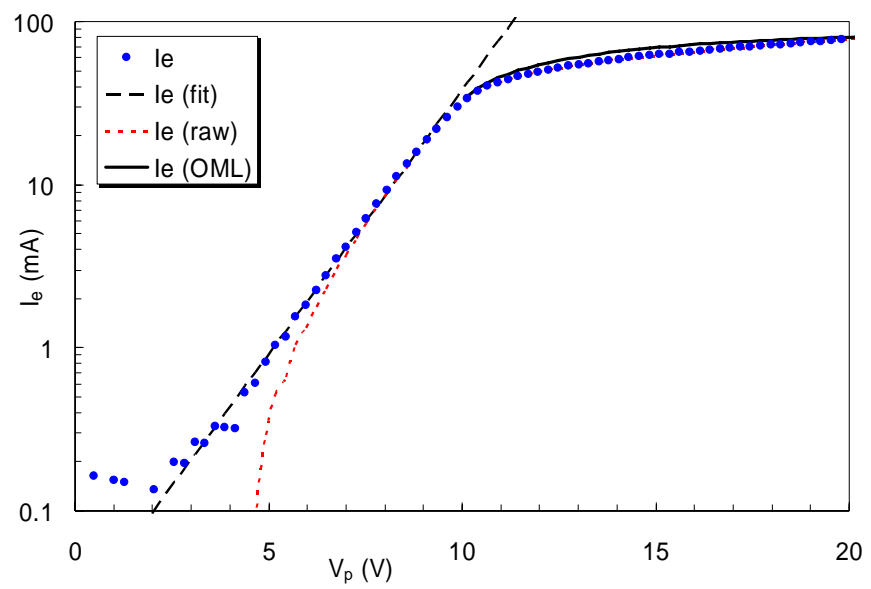

Fig. 19. Example of OML fit (-) to electron saturation current $(\bullet)$.

\section{Summary}

A linear dependence of $\mathrm{I}_{\mathrm{i}}^{2}$ on $V_{\mathrm{p}}$ indicates perfect orbiting such that ions starting far from the probe do not encounter an absorption radius or collisions that disrupt the orbits. To achieve this, a probe was designed and constructed with $R_{\mathrm{p}}$ as small as possible relative to $\lambda_{\mathrm{D}}$. Analysis of hundreds of $I-V$ curves taken at densities of industrial relevance showed that the $I_{\mathrm{i}}{ }^{2}-V_{\mathrm{p}}$ plot is almost always exactly linear. There was no evidence of the distortion of this curve by collisions, as predicted and observed at low densities of $10^{8}-10^{10} \mathrm{~cm}^{-3}$. That the simple OML formula predicting $I_{\mathrm{i}}{ }^{2} \propto V_{\mathrm{p}}$ was found to fit the experimental data is a complete surprise, since its approximations are not accurate. The exact OML formula predicts $I_{\mathrm{i}}^{2} \propto V_{\mathrm{p}}$ for high $T_{\mathrm{i}}$ or large assumed sheath radius. However, setting the OML sheath radius inside the presheath does not yield a reasonable combination of sheath thickness and $T_{\mathrm{i}}$ that would result in a linear OMLpredicted $I_{\mathrm{i}}{ }^{2}-V_{\mathrm{p}}$ plot. Nonetheless, use of the simple OML formula probably gives the density with less error than that in measuring the effective probe length. Experimental techniques for avoiding errors caused by data acquisition rate, probe heating, spurious detection of fast electrons, and plasma potential pulling were also described.

We acknowledge the support of Hiden Analytical, Ltd., in providing electronic equipment. We acknowledge the support of Hiden Analytical, Ltd., in providing electronic equipment, and of the Institute for Nuclear Energy Research, Lungtan, Taiwan. 


\section{REFERENCES}

${ }^{1}$ F.F. Chen, Phys. Plasmas 8, 3029 (2001).

2 J.D. Evans, W. Zawalsky, and F.F. Chen, Evaluation of Langmuir probe ion density measurement theories using microwave interferometry and plasma oscillation probe methods in an inductively coupled plasma, UCLA Report LTP-111 (November, 2001), unpublished.

${ }^{3}$ J.E. Allen, R.L.F. Boyd, and P. Reynolds, Proc. Phys. Soc. (London) B70, 297 (1957).

${ }^{4}$ F.F. Chen, Electric Probes, in "Plasma Diagnostic Techniques", ed. by R.H. Huddlestone and S.L. Leonard (Academic Press, New York), Chap. 4, pp. 113-200 (1965).

${ }^{5}$ I.B. Bernstein and I. N. Rabinowitz, Phys. Fluids 2, 112 (1959).

${ }^{6}$ J.G. Laframboise, Univ. Toronto Inst. Aerospace Studies Rept. 100 (June, 1966), unpublished, but results have been summarized by Chen (Refs. 4, 1).

${ }^{7}$ Y.S. Chou, L. Talbot, and D.R. Willis, Phys. Fluids 9, 2150 (1966).

${ }^{8}$ Z. Zakrzewski and T. Kopiczynski, Plasma Phys. (now Plasma Phys. Control. Fusion) 16, 1195 (1974).

${ }^{9}$ M. Tichý, M. Šicha, P. David, and T. David, Contrib. Plasma Phys. 34, 59 (1994).

${ }^{10}$ D. Trunec, P. Španěl, and D. Smith, Contrib. Plasma Phys. 35, 203 (1995).

${ }^{11}$ Z. Sternovsky, S. Robertson, and M. Lampe, Phys. Plasmas 10, 300 (2003).

${ }^{12}$ Z. Sternovsky, S. Robertson, and M. Lampe, J. Appl. Phys. 94, 1374 (2003).

${ }^{13}$ F. Iza and J.K. Lee, J. Vac. Sci. Technol A 24, 1366 (2006).

${ }^{14}$ M. Lampe, V. Gavrishchaka, G. Ganguli, and G. Joyce, Phys. Rev. Lett. 86, 5378 (2001).

${ }^{15}$ V. Pletnev and J.G. Laframboise, Phys. Plasmas 13, 073503 (2006).

${ }^{16}$ I.H. Hutchinson and L. Patacchini, Phys. Plasmas 14, 013505 (2007).

${ }^{17}$ F.F. Chen and H. Torreblanca, Plasma Phys. Control. Fusion 49, A81 (2007).

${ }^{18}$ H.M. Mott-Smith and I. Langmuir, Phys. Rev.28, 727 (1926).

${ }^{19}$ N. Hershkowitz, in Plasma Diagnostics, Vol. 1, ed. by O. Auciello and D.O. Flamm (Academic Press, New York, 1989), Chap. 3.

${ }^{20}$ F.F. Chen, Plasma Phys. Control. Fusion 39, 1533 (1997).

${ }^{21}$ I.D. Sudit and F.F. Chen, Plasma Sources Sci. Technol. 3, 162 (1994).

${ }^{22}$ F.F. Chen, Plasma Sources Sci. Technol. 15, 773 (2006).

${ }^{23}$ J.L. Jauberteau and I. Jauberteau, Plasma Sources Sci. Technol. 17, 015019 (2008).

${ }^{24}$ A.W. Molvik, A.R. Ellingboe, and T.D. Rognlien, Phys. Rev. Lett. 79, 233 (1997).

${ }^{25}$ Plasma Phys. Control. Fusion 39, 1533 (1997).

${ }^{26}$ L.S. Pilling and D.A. Carnegie, Plasma Sources Sci. Technol. 16, 570 (2007).

${ }^{27}$ F.F. Chen, Bulletin of the American Physical Society (2005) for the Gaseous Electronics Conference, San Jose, CA, October 17, 2005, paper EM2.00001.

28 J. E. Allen, Physica Scripta 45, 497 (1992).

${ }^{29}$ F.F. Chen and D. Arnush, Phys. Plasmas 8, 5051 (2001). 